\title{
Contextualizing the Relevance of Antimicrobial Resistance in the Drug Prescription and Consumption Pattern of a Tertiary Level Hospital
}

\author{
B. Bhardwaj ${ }^{1, *}$, A. Mahajan ${ }^{2}$, M. Singh ${ }^{3}$, R. Roy $^{4}$ and R. Sharma ${ }^{5}$ \\ Department of Biosciences, Manipal University Jaipur, Rajasthan-303007, India \\ "E-mail: bhrigu096@gmail.com
}

\section{ARTICLE INFORMATION}

Received: August 16, 2018

Revised: September. 05, 2018

Accepted: September 22, 2018

Published online: Oct. 08, 2018

Keywords:

Antimicrobial Resistance, Antimicrobial Stewardship, Antimicrobial Consumption, Defined Daily Dose, Intensive Care Units

\begin{abstract}
Rampant, irrational and unscientific use of antimicrobials by the healthcare practitioners is adding to the problem of antimicrobial resistance. Therefore, it is of utmost importance to evolve and implement new policies, recommence research actions and pursue steps to manage the crisis of antimicrobial resistance, which can be accomplished by a well-designed Antimicrobial Stewardship programme. A prospective observational study aiming to investigate the use of antimicrobials in Intensive Care Units by means of an AMS programme was carried out in a 400 bedded tertiary level hospital in New Delhi with 140 ICU beds. This evidence-based study looks into the prescription and consumption of antimicrobials, specifically in the ICUs, and attempts to corelate it with the resistance pattern of antimicrobials being used. All the patients being treated with antimicrobials, admitted in the ICUs of the hospital were selected for this descriptive research. Information associated with antimicrobials including the susceptibility pattern of isolates and full dosage regime of each patient from the group was obtained by conducting daily audits for a period of five months. Among the usage of different antimicrobial categories (antibacterial, antifungal and antiviral), the resistance percentage correlation was predominantly focused on the antibacterial. However, the study suggests no conclusive direct or indirect correlation between the usage of an antibacterial (in terms of prescription and consumption) and its resistance, which itself could be a cause of concern for medical practitioners.
\end{abstract}

\section{Introduction}

From the time when first antibiotic, Penicillin, was discovered in 1928 by Alexander Fleming, there had been detection and recognition of antimicrobial resistance (AMR) (Antibiotic / Antimicrobial Resistance, CDC). World Health Organization (WHO) describes AMR as "the ability of a microorganism to stop an antimicrobial from working against it" ensuing ineptness of usual treatments (Antimicrobial Resistance, WHO). In effect, microorganisms will constantly attempt to tolerate new antimicrobials and share their resistance amongst other microorganisms, which possibly will further result in persistence of infections and dispersal to others (Antibiotic / Antimicrobial Resistance, CDC). Due to this, AMR has become a chief concern in the public health sector, resulting in prodigious loss of lives and capital (Laxminarayan, 2014). In developing countries like India, there are disproportionately higher occurrences of inapt antimicrobial usage and greater levels of resistance when equated to developed countries
(Lim, 2016). Resistant strains of microorganisms are cumulating at a distressing degree, yet there is a derisory development of new antimicrobials. Progression of AMR is further encouraged by redundant and irrational usage of antimicrobials in healthcare institutions. Thus, there is a tenacious requirement for coordinated efforts to implement new policies, recommence research exertions and pursue steps to manage the crisis of AMR (Spellberg, 2014), which can be accomplished to some extent by a well-designed Antimicrobial Stewardship (AMS) programme.

According to WHO, AMS refers to "interventions designed to promote the optimal use of antibiotic agents, including drug choice, dosing, route, and duration of administration" (Antimicrobial Stewardship: A competency-based approach, WHO). The aim of this research is to investigate the use of antimicrobials in Intensive Care Units (ICUs) by means of an AMS programme. Hypothesis for the purpose of this research is - "the resistance percentage of an antimicrobial is inversely proportional to the number of times it is prescribed by the doctors in the hospital". This evidence- 
based study looks into the prescription and consumption of antimicrobials specifically in the ICUs and attempts to corelate it with the resistance pattern of antibacterial being used. The study discloses the quantity (in grams) of antimicrobial prescribed in the ICUs by substantial universal measures, which includes Defined Daily Dose (DDD) and Days of Therapy (DOT). It also focuses on antimicrobial prescription pattern of healthcare doctors, including empirical therapy used. WHO Collaborating Centre for Drug Statistics Methodology defines DDD as "the assumed average maintenance dose per day for a drug used for its main indication in adults". DDD essentially does not suggest the therapeutic dose for individual patients due to differences in their pharmacokinetic traits and severity of the ailment. It is purely used as a measurement unit (Definition and general Considerations - WHOCC, 2018). Another important unit of measurement is DOT. It refers to "the number of days during which a patient is being treated with an antimicrobial" (Griffith, 2012).

\section{Methodology}

\subsection{Study Design}

This is a prospective observational research study carried out in the ICUs of a Joint Commission International (JCI) and National Accreditation Board for Hospitals and Healthcare Providers (NABH) accredited 400 bedded tertiary level hospital in New Delhi (India). Population chosen for this study included all the patients being treated with antimicrobials, who were admitted in the Medical ICU, Pediatric ICU, Cardiac ICU and Neurological ICU of the hospital. Prior approval was obtained from the Ethical Committee of the host institution and identity of patients and treating physician was not disclosed in the study for confidential reasons.

\subsection{Data Collection}

Data was collected from August 2018 till December 2018 by conducting systematic daily audits using Google Forms. The data was extracted from patient's Medication Administration Records (MAR), progress notes, patient transfer records, Hospital Management System (HMS), hospital antibiogram and "Justification Form for Restricted Antimicrobials".

To ensure the prudent use of antimicrobials and reduce the risk of spreading resistance amongst antimicrobial agents, host institution's drug formulary designated fifteen antimicrobial agents (including meropenem, imipenem, ertapenem, doripenem, vancomycin, linezolid, colistimethate sodium, tigecycline, amphotericin B, voriconazole, teicoplanin, polymyxin B, caspofungin, fosfomycin and minocyclineas) as 'Restricted Antimicrobials'. To observe and regulate the consumption of 'Restricted Antimicrobials', a 'Justification Form for Restricted Antimicrobials' (J.F.) was created in 2016, as a "pre-prescription approach". Antimicrobial prescribing staff was required to fill the J.F. prior to prescribing any restricted antimicrobial, along with justification. Signatures of the prescribing doctor, nursing in charge, a microbiologist and a clinical pharmacist were also required on the form.

Information obtained for this study included demographic profile of the patient, primary diagnosis, initial antimicrobials being administered to the patient, full dosage regime (including, dosage form, dose, route of administration and frequency), duration of the antimicrobial treatment, change in antimicrobial prescription during the stay in the hospital, whether the antimicrobial being prescribed is restricted or not, compliance with the 'Justification Form for Restricted Antimicrobials', antimicrobial resistance pattern, duration of patient's stay and clinical outcome of the patient.

\subsection{Data Analysis}

The data collected was retrieved from google spreadsheet and saved as Microsoft Excel file. Collected data was further summarized and analysed using Pivot Table. Electronic figures and graphs of analysed data were created using 'Charts' feature of Excel. Descriptive statistical figures were expressed as the mean \pm standard deviation or as percentages.

Antimicrobial resistance pattern was obtained from the periodic summary of antimicrobial susceptibilities of local bacterial isolates, issued by the hospital's clinical microbiology laboratory. For this study the phrase 'no. of times prescribed' is calculated by the total number of patients it has been prescribed to. The re-prescription for a patient has only been counted in case of change in dosage or root of administration of that drug. Total antimicrobial class consumption (in grams) was calculated by adding all the antimicrobial prescribed in the ICUs during the study period. Defined Daily Dose (DDD) per 100 bed days was calculated as per WHO Anatomical Therapeutic Chemical (ATC) classification (ATC/DDD Index, 2019), where, a bed day refers to 'a day during which a person is confined to a bed and in which the patient stays overnight in a hospital' (DDD Indicators, WHO). Average Days of Therapy (DOT) was also calculated, where 1 DOT is denoted by any dose of an antimicrobial that is received during a period of 24 hours (Metrics and Evaluation, Public Health Ontario).

\section{Results}

A total of 843 patients were evaluated for this study, out of which 475 (56.35\%) were male and 368 (43.65\%) were 
female. 69 (8.19\%) patients were 16 years old or below and were not considered for calculating DDDs and DOT. The mean age of patients was $55.48 \pm 22.8$. During the research extent $819(97.15 \%)$ patients were admitted once, $21(2.49 \%)$ patients were admitted twice and $3(0.36 \%)$ patients were admitted thrice.

Average duration of patient's hospitalization was 8.77 \pm 5.37 days. For this parameter, 34 patients out of the total 843 patients were not assessed as their duration of hospitalization was greater than one month and they were considered as outliers. Mode for 809 patients' duration of hospitalization was 5 days.

\subsection{Antimicrobial Consumption}

Figure 1 depicts the percentage of different antimicrobial types used in the ICUs of the host institution. Antibacterial were prescribed 1631 (93.84\%) times, antifungals were prescribed $83(4.78 \%)$ times and antiviral were prescribed only $24(1.38 \%)$ times.

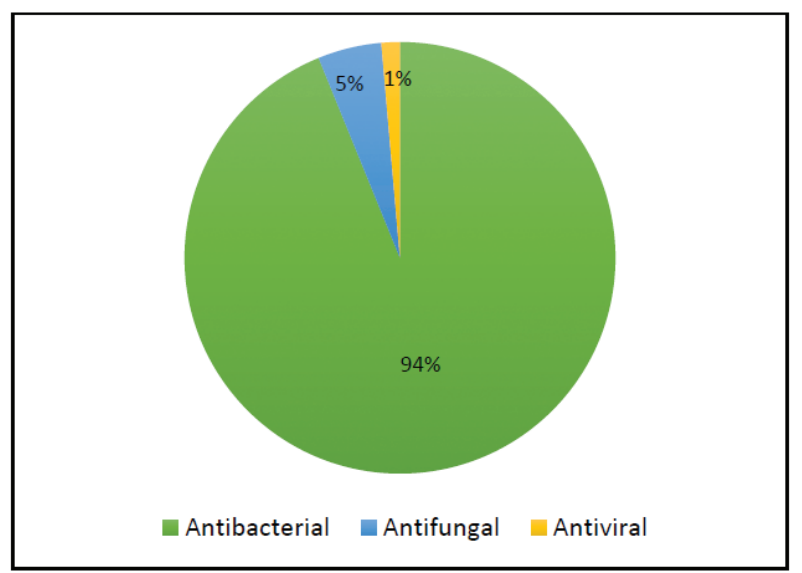

Figure 1: Antimicrobials (\%) Prescribed in ICUs.

The total usage of different antibacterial in the ICUs is portrayed in Figure 2. Among the most prescribed antibacterial classes, Cephalosporins ranked first at 32.86\% followed by Penicillin at $18.70 \%$ and Carbapenems at $10.55 \%$. The least prescribed antimicrobials were phosphonic acid derivatives and Sulfonamides at $0.06 \%$.

Figure 3 and 4 depicts the percentage of antifungal and antiviral usage in ICUs respectively. The most used antifungal was Fluconazole at $70 \%$, while the most used antiviral was Oseltamivir at 54\%.

$50 \%$ of the total patients were administered with a maximum of one antimicrobial at a time, $31 \%$ patients received 2 antimicrobials at a time, $13 \%$ patients received 3 and $6 \%$ patients were administered with 4 or more antimicrobials at a time, as shown in Figure 5.

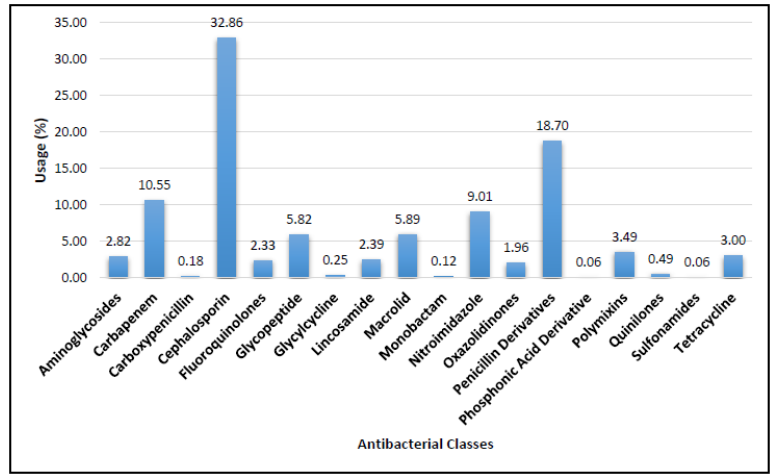

Figure 2: Antibacterial Usage (\%) in ICUs

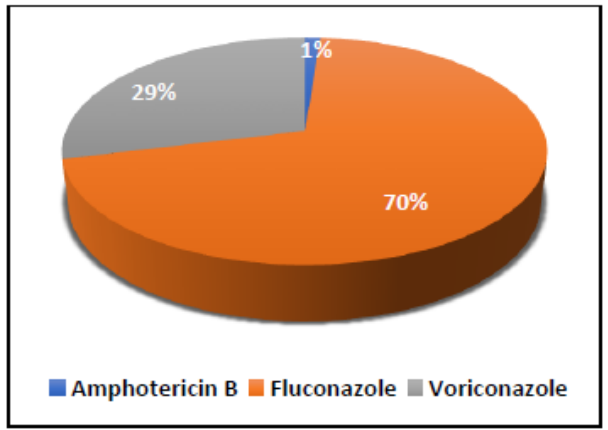

Figure 3: Antifungal Usage (\%) in ICUs

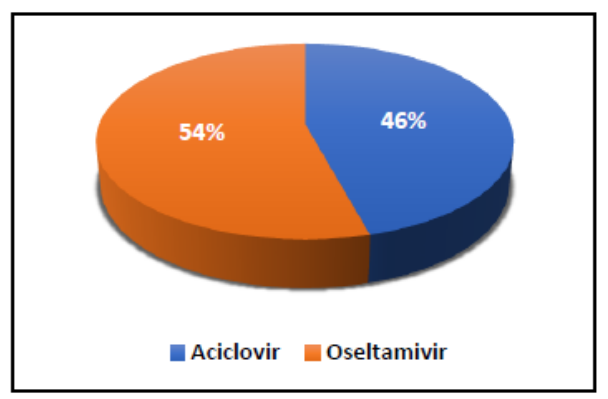

Figure 4: Antiviral Usage (\%) in ICUs

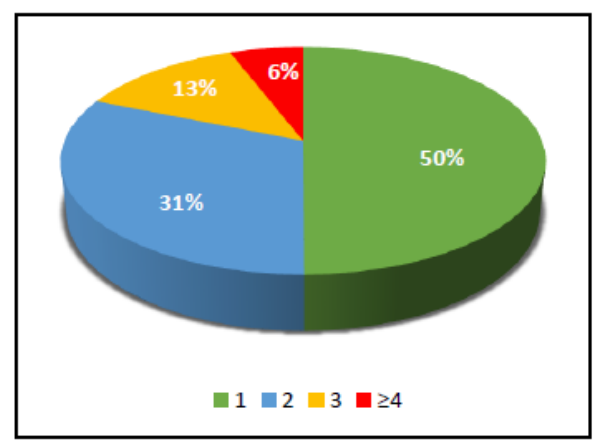

Figure 5: Maximum Antimicrobials Given at a Time 
Figure 6 shows the total number of antimicrobials prescribed to a patient during their course of stay in the hospital. 372 patients were prescribed with only a single antimicrobial, 260 patients were prescribed with 2 antimicrobials, 114 patients with 3 antimicrobials, 54 patients with 4 antimicrobials and 43 patients were prescribed with 5 or more antimicrobials.

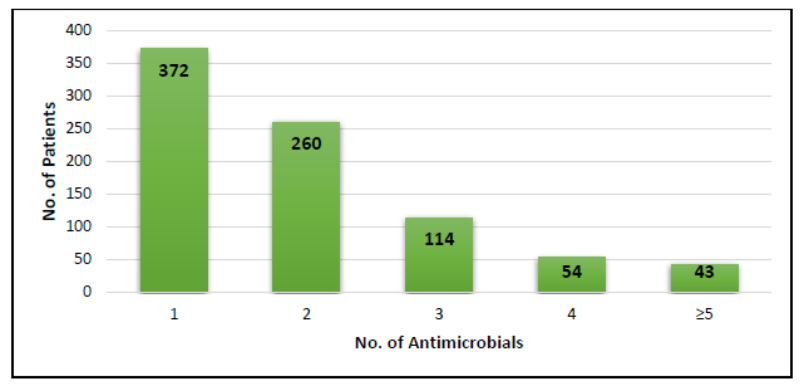

Figure 6: Total Number of Antimicrobials Prescribed to a Patient

\subsection{Restricted Antimicrobials}

238 (28.23\%) ICU patients were prescribed the 'Restricted Antimicrobials', while $605(71.77 \%)$ patients were not. Figure 7 shows the usage of Restricted Antimicrobials in the ICUs in percentage. Meropenem was the most used Restricted Antimicrobials at $44.72 \%$ followed by Teicoplanin at $23.33 \%$ and Colistin at $12.50 \%$.

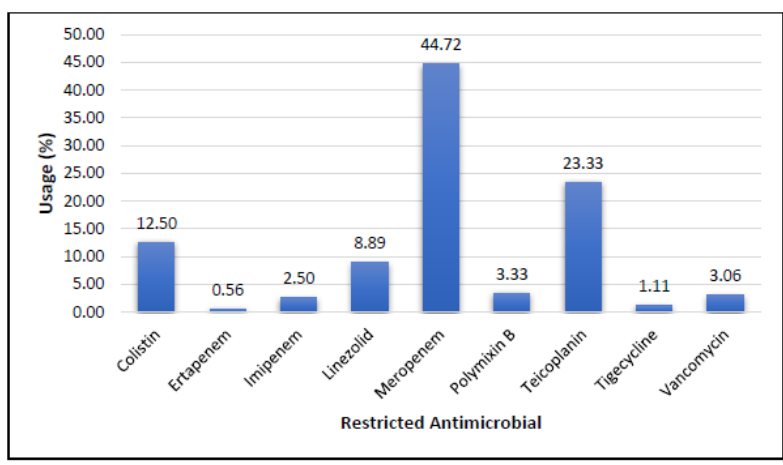

Figure 7: Restricted Antimicrobial Usage (\%)

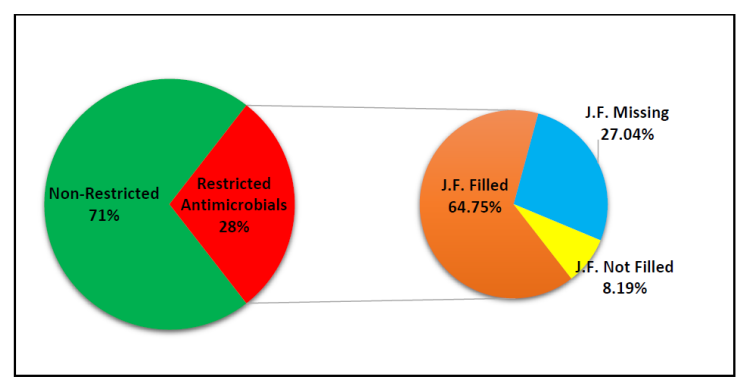

Figure 8: Compliance with 'Justification Form for Restricted Antimicrobials' (J.F.)
Figure 8 portrays that of all the $28 \%$ patients prescribed with 'Restricted Antimicrobials', J.F. was filled for $64.75 \%$ patients as per the hospital's guidelines and not filled for $8.19 \%$ patients. During the audits, it was also observed that J.F. was missing from the medical files of $27.04 \%$ ICU patients.

Table 1: Defined daily Dose (DDD) per 100 bed days

\begin{tabular}{|c|c|c|c|c|}
\hline $\begin{array}{l}\text { Antimicrobial } \\
\text { Name }\end{array}$ & $\begin{array}{l}\text { Consumption } \\
\text { (in Grams) }\end{array}$ & $\begin{array}{l}\text { WHO } \\
\text { DDD } \\
\text { (in } \\
\text { Grams) }\end{array}$ & $\begin{array}{l}\text { No. of } \\
\text { DDD } \\
\text { for adult } \\
\text { patients }\end{array}$ & $\begin{array}{l}\text { DDD per } \\
100 \text { bed } \\
\text { days }\end{array}$ \\
\hline Acyclovir & 65.70 & 4 & 16.43 & 2.12 \\
\hline Amikacin & 246.15 & 1 & 246.15 & 31.80 \\
\hline Amoxicillin (IV) & 3179.30 & 3 & 1059.77 & 136.92 \\
\hline Amoxicillin (Oral) & 71.25 & 1.5 & 47.50 & 6.14 \\
\hline Amphotericin B & 0.35 & 0.035 & 10.00 & 1.29 \\
\hline Azithromycin (IV) & 9.00 & 0.5 & 18.00 & 2.33 \\
\hline $\begin{array}{l}\text { Azithromycin } \\
\text { (Oral) }\end{array}$ & 28.70 & 0.3 & 95.67 & 12.36 \\
\hline Aztreonam & 83.00 & 4 & 20.75 & 2.68 \\
\hline Cefepime & 1314.80 & 4 & 328.70 & 42.47 \\
\hline Cefipirome & 1929.00 & 4 & 482.25 & 62.31 \\
\hline Cefoperazone & 88.00 & 4 & 22.00 & 2.84 \\
\hline Cefotaxime & 59.00 & 4 & 14.75 & 1.91 \\
\hline $\begin{array}{l}\text { Cefpodoxime } \\
\text { (Oral) }\end{array}$ & 3.60 & 0.4 & 9.00 & 1.16 \\
\hline Ceftazidime & 355.88 & 4 & 88.97 & 11.49 \\
\hline Ceftriaxone & 5800.00 & 2 & 2900.00 & 374.68 \\
\hline Cefuroxime (IV) & 183.00 & 3 & 61.00 & 7.88 \\
\hline Cefuroxime (Oral) & 62.00 & 0.5 & 124.00 & 16.02 \\
\hline $\begin{array}{l}\text { Cefuroxime + } \\
\text { Sulbactum }\end{array}$ & 724.50 & 3 & 241.50 & 31.20 \\
\hline Ciprofloxacin (IV) & 37.80 & 0.8 & 47.25 & 6.10 \\
\hline $\begin{array}{l}\text { Ciprofloxacin } \\
\text { (Oral) }\end{array}$ & 10.00 & 1 & 10.00 & 1.29 \\
\hline $\begin{array}{l}\text { Clarithromycin } \\
\text { (IV) }\end{array}$ & 304.12 & 1 & 304.12 & 39.29 \\
\hline $\begin{array}{l}\text { Clarithromycin } \\
\text { (Oral) }\end{array}$ & 288.90 & 0.5 & 577.80 & 74.65 \\
\hline Clindamycin (IV) & 336.90 & 1.8 & 187.17 & 24.18 \\
\hline $\begin{array}{l}\text { Clindamycin } \\
\text { (Oral) }\end{array}$ & 14.40 & 1.2 & 12.00 & 1.55 \\
\hline $\begin{array}{l}\text { Colistimethate } \\
\text { Sodium }\end{array}$ & 37.76 & 0.96 & 39.33 & 5.08 \\
\hline Colistin & 106.32 & 0.96 & 110.75 & 14.31 \\
\hline
\end{tabular}




\begin{tabular}{|c|c|c|c|c|}
\hline Doxycycline (IV) & 84.90 & 0.1 & 849.00 & 109.69 \\
\hline $\begin{array}{l}\text { Doxycycline } \\
\text { (Oral) }\end{array}$ & 17.20 & 0.1 & 172.00 & 22.22 \\
\hline Ertapenem & 18.00 & 1 & 18.00 & 2.33 \\
\hline Flucloxacillin & 111.00 & 2 & 55.50 & 7.17 \\
\hline Fluconazole (IV) & 132.80 & 0.2 & 664.00 & 85.79 \\
\hline $\begin{array}{l}\text { Fluconazole } \\
\text { (Oral) }\end{array}$ & 33.15 & 0.2 & 165.75 & 21.41 \\
\hline Fosfomycin & 132.00 & 8 & 16.50 & 2.13 \\
\hline Imipenem & 138.00 & 2 & 69.00 & 8.91 \\
\hline Levofloxacine & 77.50 & 0.5 & 155.00 & 20.03 \\
\hline Linezolid (IV) & 269.40 & 1.2 & 224.50 & 29.01 \\
\hline Linezolid (Oral) & 120.00 & 1.2 & 100.00 & 12.92 \\
\hline Meropenem & 3446.45 & 3 & 1148.82 & 148.43 \\
\hline $\begin{array}{l}\text { Metronidazole } \\
\text { (IV) }\end{array}$ & 1884.05 & 1.5 & 1256.03 & 162.28 \\
\hline $\begin{array}{l}\text { Metronidazole } \\
\text { (Oral) }\end{array}$ & 24.00 & 2 & 12.00 & 1.55 \\
\hline Minocycline & 0.80 & 0.2 & 4.00 & 0.52 \\
\hline Moxifloxacine & 14.80 & 0.2 & 74.00 & 9.56 \\
\hline Ofloxacin & 18.00 & 0.4 & 45.00 & 5.81 \\
\hline Ornidazole & 23.00 & 1 & 23.00 & 2.97 \\
\hline Oseltamivir (Oral) & 11.55 & 0.15 & 77.00 & 9.95 \\
\hline Piperacillin & 12676.50 & 14 & 905.46 & 116.99 \\
\hline Polymixin B & 19.30 & 0.15 & 128.67 & 16.62 \\
\hline Streptomycin & 11.00 & 1 & 11.00 & 1.42 \\
\hline Teicoplanin & 269.00 & 0.4 & 672.50 & 86.89 \\
\hline Ticarcillin & 414.20 & 15 & 27.61 & 3.57 \\
\hline Tigecycline & 2.90 & 0.1 & 29.00 & 3.75 \\
\hline Tobramycin (IV) & 0.45 & 0.24 & 1.88 & 0.24 \\
\hline $\begin{array}{l}\text { Tobramycin } \\
\text { (Inhalation) }\end{array}$ & 20.40 & 0.3 & 68.00 & 8.79 \\
\hline $\begin{array}{l}\text { Trimethoprim / } \\
\text { sulfamethoxazole }\end{array}$ & 3.84 & 0.2 & 19.20 & 2.48 \\
\hline Vancomycin & 84.29 & 2 & 42.15 & 5.45 \\
\hline Voriconazole (IV) & 12.60 & 0.4 & 31.50 & 4.07 \\
\hline $\begin{array}{l}\text { Voriconazole } \\
\text { (Oral) }\end{array}$ & 80.60 & 0.4 & 201.50 & 26.03 \\
\hline
\end{tabular}

\subsection{Defined Daily Dose (DDD)}

Total number of adult patients evaluated for this study were 774 excluding 69 pediatric patients and total number of adult patient bed days were 8170 . The DDD per 100 bed days of every antimicrobial prescribed in the ICUs during the study

period, is depicted in Table 1. The highest DDD/100 bed days was of ceftriaxone at 374.68 DDDs, whereas lowest was of tobramycin (Intravenous - IV) at 0.24 DDDs.

\subsection{Resistance Percentage of Antimicrobial corresponding to Usage Variables}

Table 2 lays down the details of the most used antimicrobials in the ICU in relation to their resistance percentage, prescription, along with their average DOT. Here, 100 resistance percentage of an antimicrobial means that all the microorganisms isolated from the various clinical samples were resistant to that particular antimicrobial. While, 0 resistance percentage of an antimicrobial indicates that none of the microorganisms isolated were resistant to that specific antimicrobial. The isolates obtained were tested for antimicrobial susceptibility using Vitek 2 systems, as per the Clinical and Laboratory Standards Institute (CLSI) guidelines.

Table 2: Resistance Percentage of Antimicrobial Corresponding to Usage Variables

\begin{tabular}{|l|l|l|l|l|}
\hline Antimicrobial & $\begin{array}{l}\text { Resistance } \\
\text { Percentage }\end{array}$ & $\begin{array}{l}\text { No. of } \\
\text { Times } \\
\text { Prescribed }\end{array}$ & $\begin{array}{l}\text { Sum of } \\
\text { Antimicrobial } \\
\text { Therapy Days }\end{array}$ & $\begin{array}{l}\text { Average } \\
\text { DOT }\end{array}$ \\
\hline Amikacin & 51.00 & 40 & 311 & 7.78 \\
\hline Amoxicillin & 75.38 & 182 & 1054 & 5.79 \\
\hline Azithromycin & 0.00 & 21 & 100 & 4.76 \\
\hline Aztreonam & 98.00 & 2 & 18 & 9.00 \\
\hline Cefepime & 67.53 & 34 & 343 & 10.09 \\
\hline Cefixime & 0.00 & 1 & 2 & 2.00 \\
\hline Cefoperazone & 61.20 & 4 & 27 & 6.75 \\
\hline Cefotaxime & 92.00 & 6 & 28 & 4.67 \\
\hline Ceftazidime & 71.83 & 14 & 116 & 8.29 \\
\hline Ceftriaxone & 76.43 & 384 & 2626 & 6.84 \\
\hline Cefuroxime & 91.75 & 14 & 134 & 9.57 \\
\hline Ciprofloxacin & 82.10 & 9 & 79 & 8.78 \\
\hline Clindamycin & 75.50 & 39 & 307 & 7.87 \\
\hline Colistin & 0.00 & 37 & 352 & 9.51 \\
\hline Ertapenem & 53.38 & 2 & 18 & 9.00 \\
\hline Imipenem & 57.07 & 9 & 92 & 10.22 \\
\hline Linezolid & 0.00 & 32 & 362 & 11.31 \\
\hline Meropenem & 58.07 & 161 & 1472 & 9.14 \\
\hline Minocycline & 100.00 & 1 & 4 & 4.00 \\
\hline Piperacillin & 77.13 & 117 & 1213 & 10.37 \\
\hline Ticarcillin & 92.25 & 3 & 57 & 19.00 \\
\hline Tigecycline & 59.50 & 4 & 38 & 9.50 \\
\hline Vancomycin & 0.00 & 11 & 111 & 10.09 \\
\hline & & & & \\
\hline
\end{tabular}

\subsection{Clinical Outcome of Patients}

As revealed in Figure 9, out of the total population 725 (86\%) patients were discharged from the hospital, while 
118 (14\%) patients expired. Data of discharged patients also include patients that left the hospital against the medical advice (LAMA patients).

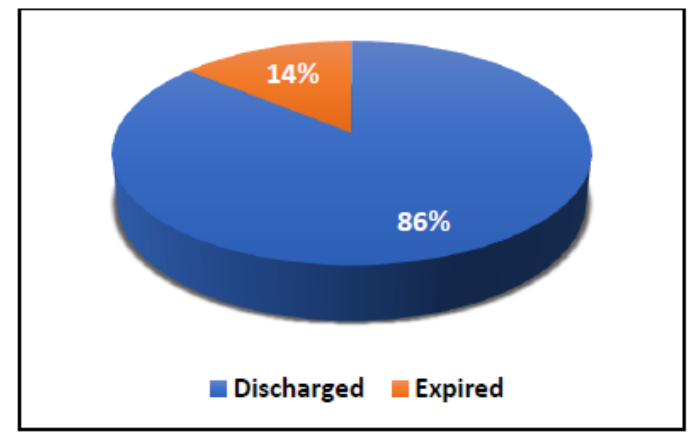

Figure 9: Clinical Outcome (\%) of the Patient

\section{Discussion}

This investigation is predominantly focused on the ICUs because ICUs are the most frequently identified source of resistant strains and hospital acquired infections (HAI) with several fold higher infection rates than general hospital wards (Jamshidi, 2009). Hence probing the ICUs will provide a comprehensive spectrum of resistance patterns and also the problems generated by it. Assessment of antimicrobial utilization pattern in ICUs is vital since it contributes to rational use of antimicrobials by distinguishing initial signs of unreasonable use of antimicrobial and it further helps to recognize appropriate interventions that are needed (Marschner, 1994).

The average days a patient was admitted in the hospital were $8.77 \pm 5.37$. This length of stay was reasonable for ICUs' patients when compared to other research studies (Sulieman, 2018) (Merzougui, 2018). Patients suffering from cardiovascular, nervous, cerebrovascular or multiple diseases usually have significantly higher length of stay. (Toptas, 2018).

All in all, the consumption of the antimicrobial, Cephalosporin was the highest among the antibacterial being used. Cephalosporin is a beta-lactam antibiotic, which is lethal to both gram positive and gram-negative bacteria, also including anaerobic (Penicillins, 2000). Such a high use of Cephalosporins might be due to the facts that it actions against a broad range of organisms, it is easy to endure, it can be administered effortlessly and it does not cost much (Alyssa, 2018). 50\% of the ICU patients were treated with a single antimicrobial while 50\% patients received multiple antimicrobial therapy. Multiple therapy prescription relies upon the type of infection and its severity. Multiple therapy might also be used in view of pharmacodynamic properties of antimicrobials, specifically synergetic and additive effect. For instance, colistin is used in combination with other antimicrobials like rifampin, $\beta$-lactams or glycopeptides, as it attributes therapeutic benefit against colistin resistant Acinetobacter baumannii (Bae, 2016).

Population of resistant pathogenic microorganisms can easily be computed by the antimicrobial consumption data for the reason that the consumption of antimicrobials is directly proportional to the progression of AMR (Sharma, 2010). Consequently, it is crucial to regulate the use of antimicrobials to reduce the risk of resistance amongst microorganisms (Austin, 1999). Correspondingly, influence of supervisory interferences and educational exercises can straightforwardly be assessed by such data.

DDD has been used as an additional benchmark of antimicrobial consumption. While DOT can be used as an additional data point for cross-referencing prescription. Calculating DDDs is extremely common and convenient techniquetoillustratetheover-allconsumptionofantimicrobials, for the reason that it authorizes equating therapeutic disparities at international, national and institutional level (Surveillance of Antimicrobial Use, WHO). Such comparisons lead to create cognizance among healthcare practitioners and allows them to identify their global position in the battle against AMR. It also permits them to incorporate improved and stringent healthcare policies and guidelines, which further assist in this fight.

Drawing from the hypothesis, according to these data points, derived in the research, there seems to be no overarching direct or indirect correlation between the variables because in certain cases (like piperacillin, ceftriaxone and amoxicillin), the prescription is high despite the resistance percentage being high. But in more than $50 \%$ cases, the correlation is indirect and the prescription number is low in instances of high percentage of resistance, like in cases of minocycline, aztreonam, ticarcillin and cefotaxime.

\section{Limitations}

Although the researcher tried to be as thorough in the study as possible, but there were few limitations that did not allow the study to reach its full potential. First and foremost, in the existing literature and the research studies available on the issue, direct comparison with this study was not possible because the attributes of the variables in this study were not found anywhere else. Also, being from biotechnology background, the skill set and knowledge base of the researcher in terms of clinically expertise was limited and thus in-depth clinical correlation could not be established among variables 


\section{Conclusion}

This research study, conducted in a tertiary level hospital in Delhi provided a summative and illustrated representation of the antimicrobial data collected of 843 patients. The correlations presented in this report included number crunching, drawing parallels and proportionality among various variable related to antimicrobial consumption in the ICUs. Drawing from these, the core purpose of the research was to find the correlation between the resistance percentage of the relevant antimicrobials and their corresponding usage among the entire data set.

The research was the first of its kind in the context of Delhi with regard to any hospital of this scale and size. The quantum of data collected for the purpose of this study is also unprecedented. The study revealed that cephalosporin class had maximum consumption (32.86\%) among different antibacterial drugs, fluconazole was the most consumed antifungal (70\%), while the most used antiviral was oseltamivir (54\%). Among cephalosporins, cefixime had the lowest resistant percentage $(0 \%)$. Other cephalosporins like cefepime, cephoperazone and ceftazidime had the resistance percentage of $67.53 \%, 61.20 \%$, and $71.83 \%$ respectively. A few other antimicrobials, which displayed zero resistance percentage were colistin, vancomycin and azithromycin.

Although drug resistance percentage is not the only factor influencing a drug prescription, as was confirmed by further discussions and consultations with the doctors and pharmacists. Issues like severity of infection, primary diagnosis, combination with other medicines also play a major role in prompting the doctor to prescribe a particular drug. But still it plays a crucial role, ignoring which might led to severe consequences.

The continued and extended use of these drugs with high resistance percentage might lead to increased development of MDRO which might further result in worse health conditions and high mortality rate, from where there is no turning back. Furthermore, treatment and management of patients with MDR is extremely complex for medical, and social facilities available to us. Also, the public health system in our country isn't equipped or developed enough to handle the issues of this complexity.

Even though there seems to be no conclusive direct or indirect correlation between the usage of a medicine (in terms of prescription and consumption) and its resistance. But the fact that there is no indirect correlation and the resistance percentage has no sustained impact on the prescription pattern, itself is a cause of concern, in medicine. The basic understanding of these concepts would lead everyone to the same conclusion, there should be an influence of the resistance a medicine has on its prescription and usage. The lack of this influence becomes a major point of intervention for medical practitioners. The foremost recommendation based on this result would be to keep the antibiogram or resistance percentage of any drug in mind before the prescription of that particular drug, especially in cases of prolonged dosage.

\section{Notes}

Financial Support - Nil

Conflicts of Interest - None Declared

\section{References}

Alyssa, R. (2018). Cephalosporins. UpToDate Website: https://www.uptodate.com/contents/cephalosporins

Antibiotic / Antimicrobial Resistance (AR / AMR), Centers for Disease Control and Prevention Website: https://www.cdc.gov/drugresistance/about.html

Antimicrobial Resistance, World Health Organization Website: https://www.who.int/antimicrobial-resistance/en/

Antimicrobial Stewardship: A competency-based approach, World Health Organization Website: https://openwho.org/courses/AMR-competency

ATC/DDD Index 2019, WHO Collaborating Centre for Drug Statistics Methodology Website: http://www.whocc.no/atcddd/

Austin, D. J., Kristinsson, K. G., \& Anderson, R. M. (1999). The relationship between the volume of antimicrobial consumption in human communities and the frequency of resistance. Proceedings of the National Academy of Sciences, 96(3), 1152-1156. https://doi.org/10.1073/pnas.96.3.1152

Bae, S., Kim, M. C., Park, S. J., Kim, H. S., Sung, H., Kim, M. N., ... \& Kim, Y. S. (2016). In vitro synergistic activity of antimicrobial agents in combination against clinical isolates of colistin-resistant Acinetobacterbaumannii. Antimicrobial agents and chemotherapy, AAC-00839. DOI: https://dx.doi.org/10.1128\%2FAAC.00839-16

DDD Indicators, World Health Organization Website: https://www.who.int/medicines/regulation/ medicines-safety/toolkit_indicators/en/

Definition and General Considerations, WHO Collaborating Centre for Drug Statistics Methodology Website: www.whocc.no/ddd/definition_and_ general_considera/

Griffith, M., Postelnick, M., \& Scheetz, M. (2012). Antimicrobial stewardship programs: methods of operation and suggested outcomes. Expert review of anti-infective therapy, 10(1), 63-73. https://doi.org/10.1586/eri.11.153 
Jamshidi, M., Javadpour, S., Eftekhari, T. E., Moradi, N., \&Jomehpour, F. (2009). Antimicrobial resistance pattern among intensive care unit patients. African Journal of Microbiology Research, 3(10), 590-594.

Laxminarayan, R., Duse, A., Wattal, C., Zaidi, A. K., Wertheim, H. F., Sumpradit, N., ... \&Greko, C. (2013). Antibiotic resistance-the need for global solutions. The Lancet infectious diseases, 13(12), 10571098.

https://doi.org/10.1016/S1473-3099(13)70318-9

Lim, C., Takahashi, E., Hongsuwan, M., Wuthiekanun, V., Thamlikitkul, V., Hinjoy, S., ... \&Limmathurotsakul, D. (2016). Epidemiology and burden of multidrugresistant bacterial infection in a developing country. Elife, 5, e18082. PDF: https://cdn. elifesciences.org/articles/18082/elife-18082-v1.pdf

Marschner, J. P., Thürmann, P., Harder, S., \&Rietbrock, N. (1994). Drug utilization review on a surgical intensive care unit. International journal of clinical pharmacology and therapeutics, 32(9), 447-451. PMID: 7820326

Merzougui, L., Barhoumi, T., Guizani, T., Barhoumi, H., Hannachi, H., Turki, E., \&Majdoub, W. (2018). Les infections nosocomialesen milieu de réanimation: incidence annuelle et aspects cliniques au Service de RéanimationPolyvalente, Kairouan, Tunisie, 2014. The Pan African medical journal, 30. https://dx.doi.org/10. 11604\%2Fpamj.2018.30.143.13824

Metrics and Evaluation. Public Health Ontario: https:// www.publichealthontario.ca/fr/eRepository/ASP\%20 Metric\%20examples.pdf
Penicillins, O. (2000). Appropriate prescribing of oral betalactam antibiotics. Am Fam Physician, 62, 611-620. http://www.p-e-g.de/Research_News/10092000-6.html

Sharma, P. R., \& Barman, P. (2010). Antimicrobial consumption and impact of "Reserve antibiotic indent form" in an intensive care unit. Indian journal of pharmacology, 42(5), 297. https://doi. org/10.4103/0253-7613.70216

Spellberg, B., \& Gilbert, D. N. (2014). The future of antibiotics and resistance: a tribute to a career of leadership by John Bartlett. Clinical infectious diseases, 59(suppl_2), S71-S75. https://doi.org/10.1093/cid/ciu392

Sulieman, H., El-Mahdi, W., Awadelkareem, M., \&Nazer, L. (2018). Characteristics of Critically-Ill Patients at Two Tertiary Care Hospitals in Sudan. Sultan Qaboos University Medical Journal, 18(2), e190. https://dx.doi. org/10.18295\%2Fsqumj.2018.18.02.011

Surveillance of Antimicrobial Use, World Health Organization Website: https://www.who.int/ medicines/areas/rational_use/AMU_Surveillance/en/

Toptas, M., SengulSamanci, N., Akkoc, İ., Yucetas, E., Cebeci, E., Sen, O., ... \& Ozturk, S. (2018). Factors Affecting the Length of Stay in the Intensive Care Unit: Our Clinical Experience. BioMed research international, 2018.

https://dx.doi.org/10.1155\%2F2018\%2F9438046 